\title{
Errata
}

\section{Revascularização cerebral na doença de moyamoya}

Arq Bras Neurocir 33(3): 266-72, 2014

Onde se lê:

Carlos Rafael Silva, Juan Antonio Castro-Flores, Carlos Eduardo Roelke, Milton Hikaru Toita, Rodrigo Becco Souza, Ulisses Prado Aguiar, Vítor Barbosa, Guilherme Brasileiro Aguiar, Mario Conti, José Carlos Veiga

\section{Leia-se:}

Rafael Carlos da Silva, Juan Antonio Castro-Flores, Carlos Eduardo Roelke, Milton Hikaru Toita, Rodrigo Becco de Souza, Ulisses do Prado Aguiar, Victor Rosseto Barboza, Guilherme Brasileiro de Aguiar, Mario Luiz Marques Conti, José Carlos Esteves Veiga 